\title{
Oral Presentation VII
}

\section{Benefit of Surgery for Primary Hyperparathyroidism in the Elderly People}

\author{
${ }^{1}$ Manabu Okada, ${ }^{2}$ Takayuki Yamamoto, ${ }^{3}$ Takahisa Hiramitsu, ${ }^{4}$ Yoshihiro Tominaga \\ ${ }^{1-4}$ Department of Transplant and Endocrine Surgery, Nagoya Daini Red Cross Hospital, Nagoya, Aichi Prefecture, Japan
}

\section{BACKGROUND AND AIMS}

The number of elderly patients with primary hyperparathyroidism (PHPT) has been increasing as a result of declining birth rate and aging population in Japan. The safety and effectiveness of surgical treatment in old people with PHPT are still contentious.

\section{METHODS}

We retrospectively investigated the characteristics and postoperative course in 55 patients over 70 years of age who underwent parathyroidectomy (PTX) for PHPT at our institution from February 1988 to May 2015.

\section{RESULTS}

Forty-four of the 55 patients had comorbidities such as hypertension, diabetes, and so on. In all the cases, PTX was successfully performed and the serum levels of parathyroid hormone and calcium lowered. Neuropsychiatric symptoms also improved in 14 patients after PTX. Except two cases, no severe complication occurred after PTX: one developed aspiration pneumonitis and one needed hemodialysis for acute exacerbation in chronic kidney disease.

\section{CONCLUSION}

Active and appropriate application of PTX might contribute to improvements in the activities of daily living and quality of life in elderly patients with PHPT.

\section{Hyperparathyroidism: Spectrum of Disorder and Clinical Practice in Sabah, Borneo}

\author{
${ }^{1}$ Doreen LP Lee, ${ }^{2}$ PP Lee, ${ }^{3}$ SZ Sharif \\ ${ }^{1-3}$ Endocrine Surgical Unit, Queen Elizabeth Hospital, Kota Kinabalu, Sabah, Malaysia
}

\section{BACKGROUND AND AIMS}

Hyperparathyroidism (HPT) results in excessive parathyroid hormone (PTH) secretion due to overactivity of the parathyroid glands. Clinical manifestations, in particular overt bone disease, vary in different parts of the world. This study aims to:

- Review the spectrum of hyperparathyroidism in Sabah, Borneo.

- Evaluate outcomes of parathyroidectomy.

\section{METHODS}

A retrospective analysis of 83 parathyroidectomy patients between January 1, 2013, and November 15, 2015, was done at a tertiary hospital. The operative approach for primary hyperparathyroidism (PHPT) was unilateral exploration after localization, whereas patients with renal hyperparathyroidism (renal HPT) underwent bilateral neck exploration (Table 1).

Table 1: Characteristics of parathyroidectomy patients

\begin{tabular}{lll}
\hline Characteristics of parathyroidectomy patients, $n=83$ & PHPT, $n=16$ & Renal HPT, $n=67$ \\
\hline Male, mean age & $\mathrm{n}=4,54.5$ years & $\mathrm{n}=37,48.7$ years \\
Female, mean age & $\mathrm{n}=1257.7$ years & $\mathrm{n}=30,52.3$ years \\
Duration of dialysis & - & 7.8 years \\
Preoperative biochemistry (mean) & & \\
Serum PTH & $527.2 \mathrm{pg} / \mathrm{ml}$ & $2417.2 \mathrm{pg} / \mathrm{ml}$ \\
Serum calcium & $2.85 \mathrm{mmol} / \mathrm{L}$ & $2.56 \mathrm{mmol} / \mathrm{l}$ \\
Postop calcium (mean) & $2.21 \mathrm{mmol} / \mathrm{L}$ & $2.32 \mathrm{mmol} / \mathrm{l}$ \\
Length of stay (LOS) & 4.5 days & 7 days \\
Average largest dimension of excised gland & $18.7 \mathrm{~mm}$ & $22.5 \mathrm{~mm}$ \\
\hline
\end{tabular}




\title{
RESULTS
}

Renal osteodystrophy (68\%) based on Dexa scan showed 33 (39\%) patients with osteopenia and 24 (29\%) osteoporotic patients. Long bone and hip fractures occurred in 14 patients (16.8\%). Intractable itch was evident in $52(63 \%)$. A total of $56 \%$ of patients with PHPT developed nephrolithiasis.

A total of $90 \%$ of patients were relieved of preoperative symptomatology within 24 hours of surgery with improvement in posture, energy level, ambulation, and mood. A total of $97.6 \%$ of patients were biochemically cured of hyperparathyroidism. Two patients $(2.4 \%)$ had recurrent hypercalcemia and are undergoing further investigations. There were $2(2.4 \%)$ perioperative mortality.

\section{CONCLUSION}

Hyperparathyroidism in this region is biochemically and clinically extreme. With favorable surgical outcomes and overall improvement in the quality of life, this review proved that parathyroidectomy is the preferred therapy for symptomatic hyperparathyroidism.

\section{Application of Tc-99m Sestamibi SPECT/CT in Patients with Renal Hyperparathyroidism}

\author{
${ }^{1}$ Chun-Min Su, ${ }^{2,3}$ Hurng-Sheng Wu, ${ }^{4}$ Po-Nien Hou, ${ }^{5}$ Li-Hsun Chen, ${ }^{6}$ Min-Chang Hung \\ ${ }^{7}$ Dev-Aur Chou, ${ }^{8}$ Chien-Hua Lin, ${ }^{9}$ Yueh-Tsung Lee, ${ }^{10}$ Shi-Wei Huang, ${ }^{11}$ Min-Ho Huang \\ ${ }^{1}$ Department of Surgery, Kaohsiung Armed Forces General Hospital, Kaohsiung City, Taiwan \\ 2,4,6,7,10,11 Department of Surgery, Show Chwan Memorial Hospital, Changhua County, Taiwan \\ 3,5,8,9 Department of Nuclear Medicine, Chang-Bing Show Chwan Memorial Hospital, Changhua County, Taiwan
}

\section{BACKGROUND AND AIMS}

To evaluate the performance of Tc-99m sestamibi single-photon computed tomography (SPECT)/computed tomography (CT) images localized in the parathyroid glands in patients with renal hyperparathyroidism.

\section{METHODS}

In this retrospective study, 65 consecutive patients with renal hyperparathyroidism underwent first parathyroidectomy. The detective performances of Tc- $99 \mathrm{~m}$ sestamibi SPECT/CT for locating the parathyroid glands were evaluated. The reports of preoperative Tc-99m sestamibi SPECT/CT was considered as follows: (1) grade 1: strong positive; (2) grade 2: weak positive; and (3) grade 3: negative. In addition, the location of enhanced lesions were divided into: (1) anterior; (2) posterior; and (3) ectopic. Baseline data and clinical characteristics, laboratory data, operative time, and complications were collected.

\section{RESULTS}

In this retrospective study, 65 consecutive patients with renal hyperparathyroidism underwent first parathyroidectomy was performed. The detective performances of Tc-99m sestamibi SPECT/CT for locating the parathyroid glands were evaluated. The reports of preoperative Tc-99m sestamibi SPECT/CT was considered as follows: (1) grade 1: strong positive; (2) grade 2: weak positive; and (3) grade 3: negative. In addition, the location of enhanced lesions were divided to: (1). Anterior (2). Posterior (3). Ectopic baseline data and clinical characteristics, laboratory data, operative time, and complications were collected.

\section{CONCLUSION}

The Tc-99m sestamibi SPECT/CT provided both functional data and anatomical information and are important to correct diagnostic and surgical approach in patients with renal hyperparathyroidism undegoing primary parathyroidectomy.

\section{The Effect of Vitamin D Deficiency on Hypocalcemia after Total Thyroidectomy}

\author{
${ }^{1}$ Ismail Cem Sormaz, ${ }^{2}$ Ahmet Yalın Iscan, ${ }^{3}$ Ilker Ozgur, ${ }^{4}$ Serhat Meric, ${ }^{5}$ Fatih Tunca, ${ }^{6}$ Yasemin Giles Senyurek \\ ${ }^{1-6}$ Department of General Surgery, Istanbul Medical Faculty, Istanbul University, Istanbul, Turkey
}

\section{BACKGROUND AND AIMS}

Hypocalcemia is the most frequent complication after thyroidectomy. We aimed to compare the impact of serum vitamin D level in postoperative hypocalcemia after total thyroidectomy.

\section{METHODS}

A total of 89 consecutive patients who underwent total thyroidectomy were included in this retrospective study. Patients were divided into two groups according to preoperative vitamin D levels. In group $1(\mathrm{n}=63)$ preoperative vitamin D level was 
$<20 \mathrm{ng} / \mathrm{ml}$, and in group $2(\mathrm{n}=26)$ preoperative vitamin D level was above $20 \mathrm{ng} / \mathrm{ml}$. Groups were compared according to their age, preoperative and postoperative corrected calcium, and parathyroid hormone (PTH) levels.

\title{
RESULTS
}

The mean age was found to be $47.8 \pm 14.1$ and the female:male ratio was 70:19 in both groups. The mean preoperative PTH values was $54.8 \pm 22.6 \mathrm{pg} / \mathrm{ml}$ and the mean postoperative PTH value was $30 \pm 19 \mathrm{pg} / \mathrm{ml}$. Postoperatively PTH values were significantly lower than preoperative PTH levels. Preoperative mean corrected calcium level was $9.3 \pm 0.4 \mathrm{mg} / \mathrm{dl}$ and postoperative corrected calcium level was $8.6 \pm 0.6 \mathrm{mg} / \mathrm{dl}$. Postoperative calcium levels were significantly decreased when we compared with preoperative calcium levels.

The demographic data showed no significant difference between the two groups of patients. The mean vitamin D level in groups 1 and 2 were found to be $11.1 \pm 4$ and $30.6 \pm 10.4 \mathrm{ng} / \mathrm{ml}$ respectively. Although the preoperative serum PTH levels showed statistically significant difference between the two groups ( $58.4 \pm 24.6$ vs $45.9 \pm 13.1 \mathrm{pg} / \mathrm{ml}$ in groups 1 and 2 respectively; all were found within the normal range). Postoperative 1st day, PTH levels were found to be $31.9 \pm 20.5$ and $25.8 \pm 14.4 \mathrm{pg} / \mathrm{ml}$ in groups 1 and 2 respectively. Postoperative corrected calcium values showed no significant difference between groups 1 and $2(8.5 \pm 0.6$ vs $8.7 \pm 0.7 \mathrm{mg} / \mathrm{dl})$.

\section{CONCLUSION}

Although vitamin D deficiency is a common entity in patients undergoing total thyroidectomy, this present study failed to show any relation between preoperative vitamin D levels and postoperative hypocalcemia.

\section{Improving the Efficacy of Post-parathyroidectomy Hypocalcemia Treatment: An Evidence-based Calcium Regimen}

\author{
${ }^{1}$ Jih Huei Tan, ${ }^{2}$ Henry Chor Lip Tan, ${ }^{3}$ Seng Cheong Loke, ${ }^{4}$ Sarojah Arulanantham \\ ${ }^{1,2,4}$ Department of General Surgery, Hospital Sultan Ismail, Johor Bahru, Malaysia \\ ${ }^{3}$ Institute of Gerontology, Universiti Putra Malaysia, Selangor, Malaysia
}

\section{BACKGROUND AND AIMS}

Postoperative hypocalcemia is common after parathyroid surgery for renal hyperparathyroidism. It tends to be more severe in Malaysian population. Calcium infusion often is required to treat the hypocalcemia in addition to the oral supplement. We compared a new infusion regimen to one commonly used in Malaysia based on $2003 \mathrm{~K} /$ DOQI guidelines.

\section{METHODS}

A study was conducted in the Endocrine Surgical Unit, Hospital Sultan Ismail, Johor Bahru, Malaysia. Retrospective data on serum calcium and infusion rates was collected from 2011 to 2015. A comparison between regimens was made based on treatment efficacy (hypocalcemia duration, total infusion amount, and time) and calcium excursions (outside target range, peak and trough calcium) using bar charts and an unpaired t-test.

\section{RESULTS}

A total of 51 and 34 patients on the original and new regimens respectively were included. The new regimen had shorter period of hypocalcemia (28.9 vs 66.4 hours, $\mathrm{p}=0.04)$ and required less calcium infusion $(67.7$ vs $127.2 \mathrm{mmol}, \mathrm{p}=0.02)$ for a shorter duration (57.3 vs 102.9 hours, $\mathrm{p}=0.001$ ). Calcium excursions and peak and trough calcium were not significantly different between regimens. Early postoperative high excursions occurred when the infusion was started in spite of elevated perioperative calcium levels.

\section{CONCLUSION}

The new infusion regimen was superior to the original in that it required a shorter treatment period and resulted in less hypocalcemia. We found that early aggressive calcium replacement is unnecessary and raises the risk of rebound hypercalcemia.

\section{Preoperative Cardiac Evaluation with Dipyridamole Thallium-201 Screening reduced Perioperative Mortality and Cardiovascular Complications in Secondary Hyperparathyroidism Patients undergoing Parathyroidectomy}

\author{
${ }^{1}$ Shih-min Yin, ${ }^{2}$ Fong-Fu Chou, ${ }^{3}$ Shun-Yu Chi \\ ${ }^{1-3}$ Department of General Surgery, Kaohsiung Chang Gung Memorial Hospital, Kaohsiung City, Taiwan
}

\section{BACKGROUND AND AIMS}

In this study, we evaluated the value of a preoperative dipyridamole thallium-201 myocardial perfusion scan for reducing perioperative cardiac complications in patients with symptomatic secondary hyperparathyroidism (SHPTH) undergoing parathyroidectomy with autotransplantation. 


\section{METHODS}

We conducted a retrospective cohort study of patients with ESRD and severe SHPTH (iPTH $>800 \mathrm{pg} / \mathrm{dL}$ or iPTH >500 pg/dL with intolerable symptoms) who received a parathyroidectomy from January 2011 to October 2015. A preoperative evaluation of dipyridamole thallium-201 myocardial scan was applied for all patients, followed by coronary angiography and revascularization for those with abnormal thallium result and severe coronary arterial disease. The primary outcomes were 30-day surgical mortality, cardiovascular morbidity, and the length of hospital stay. Patients' characteristics were compared between thallium scan positive and negative groups for further evaluation of risk factors (Table 1).

\section{RESULTS}

A total of 238 patients were included for the final analysis. Positive thallium defect was observed in 18 patients (7.6\%). A clinical important lesion was revealed in 14 patients (5.9\%) after coronary angiography, and 8 patients (3.3\%) received stent implantation or balloon angioplasty during the procedure.

There was no 30-day surgical mortality in this series. The incidence of 30-day cardiac-related morbidity was $0.84 \%$ ( 2 of 238 ) that happened during and immediately after surgery, which was substantially lower than that of other series. The overall surgical success rate was $87 \%$. There was no significant difference in hospital stay length between thallium scan positive and negative groups. Positive thallium defect was significantly higher in males $(p=0.016)$ and those with higher BMI scores $(p=0.01)$ and past history of DM $(p=0.07)$ and coronary arterial disease (CAD) $(p<0.01)$.

\section{CONCLUSION}

Application of preoperative dipyridamole thallium-201 myocardial perfusion scan and following coronary revascularization may reduce the incidence of perioperative cardiac morbidity and mortality in SHPTH patients undergoing parathyroidectomy. Male patients with higher BMI score or a history of DM and CAD should consider dipyridamole thallium-201 myocardial perfusion scan as a Routine preoperative evaluation.
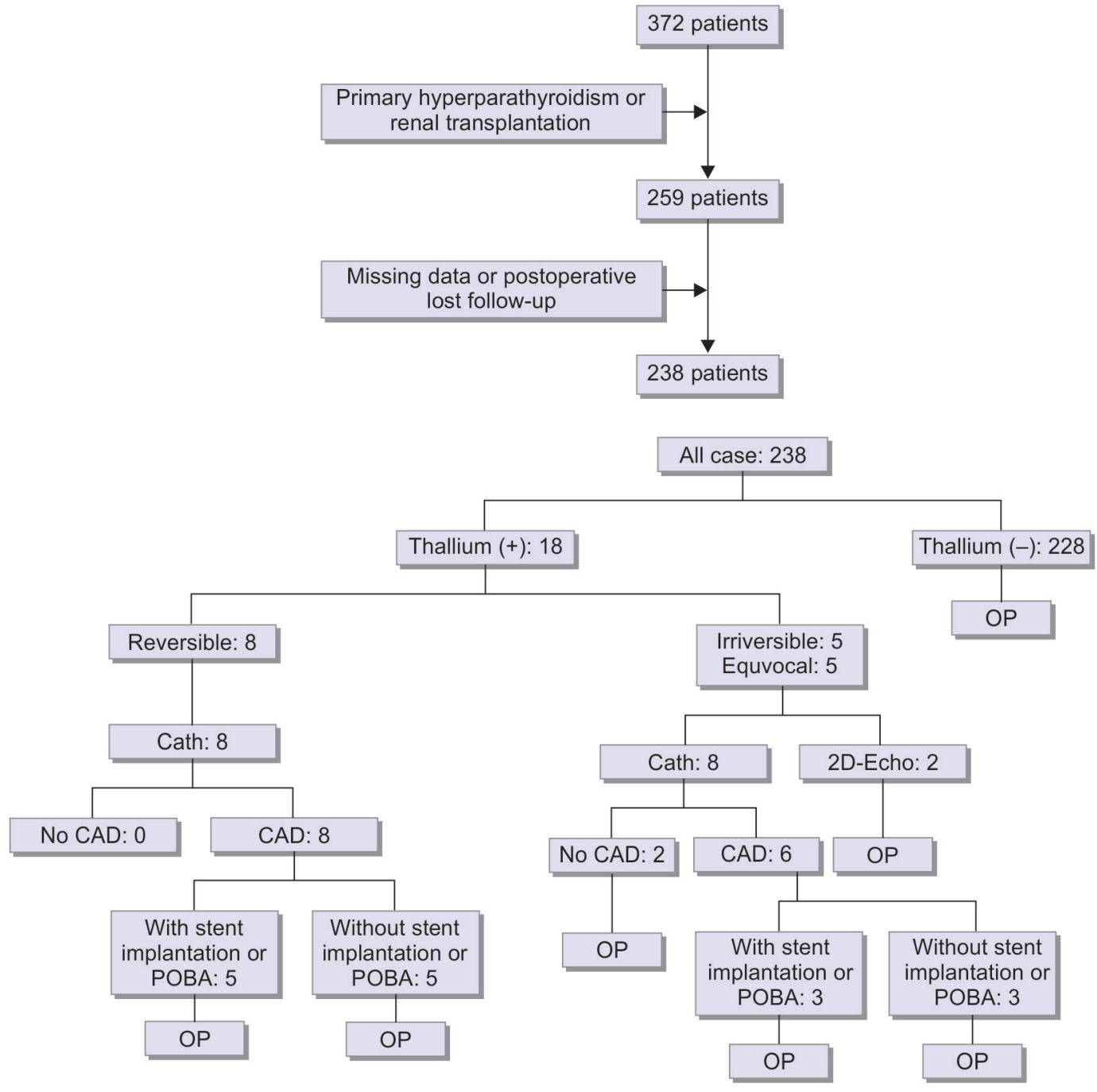
Table 1: Patient characteristics for parathyroidectomy performed for secondary hyperparathyroidism, by thallium scan result

\begin{tabular}{llllr}
\hline & Overall & Thallium scan normal & Thallium scan abnormal \\
& $N=238(100 \%)$ & $N=220(924 \%)$ & $N=18(7.6 \%)$ & $p$-value \\
\hline Age, years & $55.07 \pm 10.39$ & $54.76 \pm 10.53$ & $58.89 \pm 7.81$ & 0.105 \\
Dialysis duration, years & $9.04 \pm 4.82$ & $9.09 \pm 4.827$ & $6.94 \pm 4.33$ & 0.341 \\
BMI, kg/m2 & $23.12 \pm 3.54$ & $22.9 \pm 3.49$ & $25.81 \pm 3.69$ & 0.01 \\
Ca & $10.33 \pm 1.01$ & $10.39 \pm 0.76$ & $10.35 \pm 0.78$ & 0.612 \\
P & $5.65 \pm 1.7$ & $5.614 \pm 1.66$ & $653 \pm 1.95$ & 0.073 \\
iPTH & $1561.48 \pm 839.12$ & $1552.8 \pm 848.164$ & $1733.72 \pm 91052$ & 0.708 \\
Hospital Stay & $7.34 \pm 1.54$ & $731 \pm 1.19$ & 7.72 .12 .02 & 0.274 \\
Gender & & & & 0.016 \\
Male & $78(32.77 \%)$ & $67(30.50 \%)$ & $11(61.10 \%)$ & 0.246 \\
Female & $160(67.23 \%)$ & $153(69.50 \%)$ & $7(38.90 \%)$ & \\
Dialysis Type & & & $18(100 \%)$ & 0.045 \\
Hemodialysis & $208(87.39 \%)$ & $190(86.40 \%)$ & $0(0 \%)$ & 0.455 \\
Peritoneal dialysis & $26(10.92 \%)$ & $26(11.80 \%)$ & $0(0 \%)$ & $<0.01$ \\
Mix type & $4(1.68 \%)$ & $4(1.80 \%)$ & $5(27.80 \%)$ & $13(72.20 \%)$ \\
DM & $28(11.76 \%)$ & $23(10.50 \%)$ & $10(56 \%)$ & \\
HTN & $149(62.61 \%)$ & $136(61.80 \%)$ & $0(0.00 \%)$ & $2(0.84 \%)$ \\
CAD & $21(8.82 \%)$ & $11(5.00 \%)$ & & \\
$30-$ day Surgical Mortality & $0(0.00 \%)$ & $0(0.00 \%)$ & $0(0.00 \%)$ & \\
30-day Cardiac-related Morbidity & $0(0.00 \%)$ & &
\end{tabular}

\title{
Biochemical and Immunohistochemical Profile of Atypical Parathyroid Adenomas
}

\author{
${ }^{1}$ Roma Pradhan, ${ }^{2}$ Amit Agarwal, ${ }^{3}$ Niraj Kumari, ${ }^{4}$ Narendra Krishnani, ${ }^{5}$ Sushil Kumar Gupta \\ ${ }^{6}$ Gyan Chand, ${ }^{7}$ Anjali Mishra, ${ }^{8}$ Gaurav Agarwal, ${ }^{9}$ Ashok Kumar Verma, ${ }^{10}$ Saroj Kanta Mishra \\ 1,2,6-10Department of Endocrine Surgery, Sanjay Gandhi Postgraduate Institute of Medical Sciences, Lucknow, Uttar Pradesh, India \\ ${ }^{3,4}$ Department of Pathology, Sanjay Gandhi Postgraduate Institute of Medical Sciences, Lucknow, Uttar Pradesh, India \\ ${ }^{5}$ Department of Endocrinology, Sanjay Gandhi Postgraduate Institute of Medical Sciences, Lucknow, Uttar Pradesh, India
}

\section{BACKGROUND AND AIMS}

It is frequently difficult to determine the malignant potential of atypical parathyroid adenomas histologically; hence, the authors sought to define the molecular phenotype of atypical parathyroid adenomas.

\section{METHODS}

The histopatholgical diagnosis of atypical adenoma was made according to WHO criteria. We performed parafibromin, APC, galectin-3, and PGP9.5 expression analysis by immunohistochemistry in 25 cases of atypical adenomas. For parafibromin, the primary antibody used was mouse monoclonal antibody targeting exon 3 of human parafibromin (clone 2H1), rabbit monoclonal antibody against $\mathrm{N}$ terminal polypeptide for APC (clone EP701Y, dilution 1:50, AbCam), mouse monoclonal antibody for galectin-3 (clone B2C10, dilution 1:50, Santa Cruz), and rabbit polyclonal antibody (Dako) for PGP 9.5.

\section{RESULTS}

The clinical features and biochemistry were similar to patients of parathyroid adenomas. Because of intraoperative suspicion of parathyroid carcinoma, enbloc excision including hemi-thyroidectomy was done in six patients. Complete or partial loss of parafibromin was seen in 17/25 (68\%) patients. Loss of APC expression was seen in 9/25 (36\%). Increased expression of galectin-3 and PGP 9.5 was seen in $7 / 25(28 \%)$ and 5/25 (20\%) patients respectively. Only one atypical adenoma showed all IHC marker reactivity s/o malignancy, and its weight was $11.4 \mathrm{gm}$ (PTH: $2773 \mathrm{pg} / \mathrm{ml}$ ). None of our atypical adenomas showed evidence of recurrence or metastasis on follow-up.

\section{CONCLUSION}

Atypical adenomas may exhibit a molecular phenotype, which resembles parathyroid carcinoma especially parafibromin staining, and hence may require a closer follow-up. 


\title{
Comparison between Subtotal Parathyroidectomy and Total Parathyroidectomy with Autotransplantation for Secondary Hyperparathyroidism
}

\author{
${ }^{1}$ Murat Özdemir, ${ }^{2}$ Erkan Güler, ${ }^{3}$ Özer Makay, ${ }^{4}$ Berk Göktepe, ${ }^{5}$ Gökhan lçöz, ${ }^{6}$ Mahir Akyıldız \\ 1-3,5,6 Department of General Surgery, Division Endocrine Surgery, Ege University Hospital, Izmir, Turkey \\ ${ }^{4}$ Department of General Surgery, Çerkeş State Hospital, Çankırı, Turkey
}

\section{BACKGROUND AND AIMS}

The purpose of this study was to compare the efficacy of two surgical techniques (subtotal parathyroidectomy and total parathyroidectomy with autotransplantation) in patients with secondary hyperparathyroidism (HPT) due to chronic renal failure.

\section{METHODS}

A total of 15 patients, who underwent parathyroid surgery for secondary hyperparathyroidism, were reviewed retrospectively. The patients were divided into two groups. Subtotal parathyroidectomy had been performed in nine patients, while total parathyroidectomy with autotransplantation had been carried out in six patients. The following values were compared between groups: Intraoperative PTH measurements (quick PTH assay), serum calcium, and PTH and phosphorus levels on the first postoperative day, first month, and sixth months postoperatively. During follow-up, persistent disease, recurrence and re-operation, the need for medical treatment, and survival were evaluated.

\section{RESULTS}

A total of 15 patients were followed for a median of 38 months (6-70 months). In both groups, intraoperative PTH decreased more than $50 \%$. During follow-up, it was observed that renal transplantation had been performed in three cases. These underwent subtotal parathyroidectomy. Two patients in the subtotal parathyroidectomy group and one in the total parathyroidectomy with autotransplantation died due to non-hyperparathyroidism reasons. There was no significant difference between the two groups, regarding postoperative hypocalcemia $(p=0.435)$. Nevertheless, both surgical procedures were not statistically significant for persistent disease, recurrence, and survival $(\mathrm{p}=0.209)$.

\section{CONCLUSION}

According to our study, both surgical techniques are effective surgical options for the treatment of secondary HPT. The surgeon can determine the surgical method to be applied according to his own experiences.

\section{Short-term Outcome of Parathyroidectomy in Refractory Renal Hyperparathyroidism}

\author{
${ }^{1}$ Nur Shahida Wahab, ${ }^{2}$ Anita Baghawi, ${ }^{3}$ Sarinah Basro, ${ }^{4}$ Normayah Kitan, ${ }^{5}$ Nor Hisham Muda, ${ }^{6}$ Lisa Mohamed Nor \\ ${ }^{1-5}$ Department of Breast and Endocrine Surgery, Putrajaya Hospital, Putrajaya, Malaysia \\ ${ }^{6}$ Clinical Research Center, Putrajaya Hospital, Putrajaya, Malaysia
}

\section{BACKGROUND AND AIMS}

Parathyroid surgery, with or without autotransplantation, plays a crucial role as a treatment of refractory hyperparathyroidism. This study analyzed the short-term outcome of parathyroidectomy for renal hyperparathyroidism in our center from the year 2004 until August 2015.

\section{METHODS}

Patients who had undergone parathyroidectomy for renal hyperparathyroidism (with or without autotransplantation) at a single institution (Putrajaya Hospital) from 2004 to August 2015 were studied retrospectively. Patient characteristics, pre- and postoperative biochemical markers, intraoperative findings, and outcomes including readmission rate within 30 days, recurrence rates, postoperative morbidity, and mortality were studied.

\section{RESULTS}

A total of 366 patients had undergone parathyroidectomy for renal hyperparathyroidism within the duration of more than 10 years. All patients were on long-term dialysis ( $98.4 \%$ on hemodialysis and $1.6 \%$ on peritoneal dialysis), with a median time of 8 years from the initiation of dialysis to parathyroidectomy. A total of 330 patients had undergone parathyroidectomy alone and the remaining had autotransplantation as well in the same setting.

Postoperatively, the mean calcium was 2.258 with a standard deviation of 0.224 (compared to the mean preoperative calcium of 2.483). Of 337 patients, $82 \%$ had all four glands identified intraoperatively and confirmed histopathologically. A total of $2.1 \%$ patients were readmitted to our center within 30 days postoperatively. 
During our 2-month postop follow-up, $96.3 \%$ of the patients reported resolved bone pain. Nine patients developed recurrence, requiring re-exploration with the median time to recurrence of 1 year. Only two out of nine patients with recurrence had autotransplantation done in the first surgery.

Mortality was recorded in nine patients (3.73\%), with acute coronary syndrome and hospital-acquired pneumonia as the main causative factors (contributing $1.66 \%$ each to the mortality rate).

\title{
CONCLUSION
}

Parathyroidectomy does not only effectively lower the calcium level, but also significantly resolves the patient's symptom (bone pain) within a short period postoperatively, thus improving the patient's quality of life.

\section{Surgical Outcomes for Secondary Hyperparathyroidism: Single-institution Experience}

\author{
${ }^{1}$ Eun Jeong Ban, ${ }^{2}$ Min Jhi Kim, ${ }^{3}$ JungBum Choi, ${ }^{4}$ Seul Gi Lee, ${ }^{5}$ Taehyung Kim \\ ${ }^{6}$ Cho Rok Lee, ${ }^{7}$ Jandee Lee, ${ }^{8}$ Sang-Wook Kang, ${ }^{9}$ Jong Ju Jeong, ${ }^{10}$ Kee-Hyun Nam, ${ }^{11}$ Woong Youn Chung \\ ${ }^{1-11}$ Department of Surgery, Yonsei University College of Medicine, Seoul, South Korea
}

\section{BACKGROUND AND AIMS}

Secondary hyperparathyroidism (SHPT) is a common complication of long-term dialysis patients, and surgical parathyroidectomy (PTX) remains necessary in patients resistant to medical therapy. The aim of this study was to review our experience of surgical management of SHPT.

\section{METHODS}

We conducted a retrospective study of patients with chronic renal failure who received PTX over 10 years from January 2004 to September 2014. Surgical indication was established according to clinical or biological assessment.

\section{RESULTS}

We included 61 patients with the average age of 47.5 years, of which $27.9 \%$ were male and $72.1 \%$ female, in the dialysis for 11.42 \pm 5.18 years before PTX. The most common indication of PTX (65.6\% of the cases) was the persistence of serum PTH of more than $800 \mathrm{pg} / \mathrm{ml}$ associated with hypercalcemia and/or hyperphosphatemia refractory to medical treatment. The operations performed were: subtotal parathyroidectomy (SPTX) in 24 cases, total parathyroidectomy with autotransplantation (TPTX+AT) in 33 cases, and total parathyroidectomy (TPTX) alone in 4 cases. Histological examination of parathyroid gland specimens disclosed diffuse hyperplasia in 48 patients $(82.8 \%)$ and nodular hyperplasia in 8 patients $(13.8 \%)$. The postoperative calcium level was in the normal range in 29 cases, low comparatively to the baseline in 20 cases and high in 12 cases. The parathyroid hormone (PTH) level of more than $300 \mathrm{pg} / \mathrm{ml}$ was checked in four patients (6.56\%) 3 months postoperatively. After a mean follow-up of 40 months, three patients $(4.92 \%)$ had persistent disease.

\section{CONCLUSION}

In conclusion, PTX is very effective for the treatment of hyperparathyroidism in patients with advanced SHPT refractory to medical treatment. 